\title{
ON A CONJECTURE OF CARMICHAEL
}

\author{
V. L. KLEE, JR. ${ }^{1}$
}

Carmichael $[1]^{2}$ conjectured that for no integer $n$ can the equation $\phi(x)=n$ ( $\phi$ being Euler's totient) have exactly one solution. To support the conjecture, he showed that each $n$ for which there is a unique solution must satisfy a restriction which implies $n>10^{37}$. In this note we prove the validity of restrictions considerably stronger than those of Carmichael, and raise the lower bound on $n$ to $10^{400}$.

We shall denote by $X$ the set of all integers $x$ for which $\phi(y)=\phi(x)$ implies $y=x$. (If the conjecture is correct, $X$ is empty, and the theorems stated are vacuously satisfied.)

(1) ThEorem. Suppose that $\bar{x}=\prod_{A} p_{i}^{a_{i}}$ is in $X$, where the $p_{i}$ 's are distinct primes and $A$ is the range of the index $i$. Let $m=\prod_{B} p_{i}^{a_{i}-1}\left(p_{i}-1\right)$ - $\prod_{c} p_{i}^{c_{i}}$ where $B$ and $C$ are disjoint subsets of $A$ (one of them may be empty) and $c_{i} \leqq a_{i}-1$ for $i$ in $C$. Then if $p$ is prime and $p-1=m$, we have $p \mid \bar{x}$.

For if $p \nmid \bar{x}$, we have $\phi\left(p \cdot \prod_{A-B-c} p_{i}^{a_{i}} \cdot \prod_{c} p_{i}^{a_{i}-c_{i}}\right)=\phi(\bar{x})$, contrary to the definition of $X$.

(1.1) Corollary. Suppose, under the hypotheses of (1), that $B$ has the following property: if $q$ is prime and $q \mid\left(p_{j}-1\right)$ for some $j$ in $B$, then $q \mid \bar{x}$. We must then have $p^{2} \mid \bar{x}$.

For under this condition we have $p-1=\prod_{D} p_{i}^{d_{i}}, D$ being a subset of $A$. So if $p \mid \bar{x}$ but $p^{2} \nmid \bar{x}$, then $\phi\left(\prod_{A-D} p_{i}^{a_{i}} \cdot \prod_{D} p_{i}^{a_{i}+d_{i}} / p\right)=\phi(\bar{x})$, contrary to the definition of $X$.

(1.2) Corollary. If, in the hypotheses of (1), B is empty, we have $p^{2} \mid \bar{x}$.

(1.3) Corollary. $4 \mid \bar{x}$. If $f$ is a Fermat prime such that $f \mid \bar{x}$, then $f^{2} \mid \bar{x}$.

(1.2) and (1.3) are Carmichael's original conditions. From (1.1) and (1.3) it follows that $\bar{x}$ is divisible by $3^{2}, 7^{2}, 43^{2}, 3^{3}$ or $13^{2}, \cdots$. (By extending this list Carmichael showed both $\bar{x}$ and $\phi(\bar{x})$ to be greater than $10^{37}$.)

Presented to the Society, October 25, 1947; received by the editors December 12, 1946, and, in revised form, April 5, 1947.

${ }^{1}$ I should like to thank Professor C. G. Jaeger of Pomona College for arousing my interest in Euler's $\phi$-function.

${ }^{2}$ Numbers in brackets refer to the references at the end of the paper. 
(2) Theorem. Suppose $u=2^{a} s \prod_{A} f_{i}^{a_{i}}$ is in $X$, where the $f_{i}^{\prime}$ 's are distinct Fermat primes other than 3, and $s$ is odd and divisible by no Fermat prime other than 3. Suppose that $C \subset A, 2 \leqq c \leqq a, v=2{ }^{c} s \prod c f_{i}^{a_{i}}$. Then $v$ is in $X$ if and only if either $C=A$, or for each index $i$ in $A-C$, $2^{c} \mid\left(f_{i}-1\right)$.

Clearly if $v$ is in $X$, one of the stated conditions is valid. Now suppose that $y$ is an even integer such that $\phi(y)=\phi(v)$, and let $D$ denote the subset of $A-C$ consisting of all indices $i$ in $A-C$ for which $f_{i} \mid y$. Let $d_{i}=a_{i}-1$ for $i$ in $D, d_{i}=a_{i}$ for $i$ in $(A-C)-D$, and

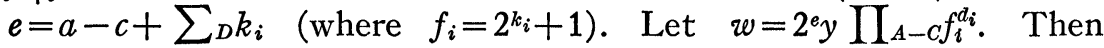
$\phi(w)=\phi(u)$. If one of the stated conditions holds, $y \neq v$ implies that either $y$ is divisible by some prime $q$ such that $q \nmid u$, or $u$ is divisible by a non-Fermat prime $p$ such that $p \nmid y$. In either case $w \neq u$, contradicting the hypothesis that $u$ is in $X$. Hence we have $y=v$ and $v$ is in $X$.

(2.1) Corollary. If $u$ is in $X$, there is an element $v$ of $X$ such that (i) $v \mid u$; (ii) $8 \nmid v$, and $v$ is divisible by no Fermat prime other than 3.

An element of $X$ which satisfies condition (ii) of (2.1) will be called a reduced element of $X$, and the collection of all reduced elements will be denoted by $R(X)$. Clearly the structure of $X$ is fairly well determined by that of $R(X)$, so henceforth we shall confine our attention to $R(X)$. If an element of $X$ has no divisor in $X$, we shall call it irreducible, and shall denote the collection of all such elements by $I(X)$.

(3) Theorem. Let $\bar{x}=\prod_{B} p_{i}^{b_{i}}$ be in $R(X)$, where the $p_{i}$ 's are distinct primes. For an integer $m$, let $B(m)$ denote the subset of $B$ consisting of all indices $i$ for which $m \mid\left(p_{i}-1\right)$. Let $w=\prod_{B-B(m)} p_{i}^{b_{i}}$. Then either $w$ is in $R(X)$ or $m \mid \phi(w)$.

Suppose $w \in R(X)$. Then $w \notin X$, and there is an integer $y \neq w$ such that $\phi(y)=\phi(w)$. And if $(y, \bar{x} / w)=1, \phi(y \bar{x} / w)=\phi(\bar{x})$, contradicting the fact that $\bar{x} \in X$. So for some $i$ in $B(m)$, we have $p_{i} \mid y$, whence $\left(p_{i}-1\right) \mid \phi(w)$. But $m \mid\left(p_{i}-1\right)$, so $m \mid \phi(w)$.

(3.1) Corollary. If, in (3), $\bar{x} \in I(X), m$ is prime, and $B(m)$ is not empty, then $m^{2} \mid \bar{x}$.

Since $B(m)$ is not empty, $w<\bar{x}$. But then, since $\bar{x} \in I(X), w \in X$. Hence $m \mid \phi(w)$, whence from the definition of $w$ it is clear that $m^{2} \mid \bar{x}$.

(3.2) Corollary. If $\bar{x} \in I(X), p$ is prime and $p \mid \bar{x}$, then $p^{2} \mid \bar{x}$.

This follows from (1.1). 
(3.3) Corollary. If $\bar{x} \in I(X), \phi(\bar{x})$ is divisible by no Fermat prime other than 3.

This is an immediate consequence of (3.1). (3.1), (3.2), and (3.3) together eliminate $11,23,31,41,47, \cdots$ as possible divisors of $\bar{x}$ or $\phi(\bar{x})$ if $\bar{x} \in I(X)$.

It would be of interest to know the relationship of $R(X)$ to $I(X)$, and of $I(X)$ to its smallest member.

We now apply (1.1) to show the following theorem. $10^{400}$

(4) Theorem. If $\bar{x}$ is in $X$, then both $\bar{x}$ and $\phi(\bar{x})$ are greater than

We know $3^{2} \mid \bar{x}$, and shall consider three possibilities: (I) $3^{3} \nmid \bar{x}$; (II) $3^{3} \mid \bar{x}$ but $3^{4} \gamma \bar{x}$; (III) $3^{4} \mid \bar{x}$. Suppose that $\bar{x}=\prod p_{i}^{a_{i}}$, the $p_{i}$ 's being distinct primes, and let $m=\prod p_{i}^{a_{i}-1}$. In each case 3,7 , and 43 are divisors of $m$.

In case (I) any prime of the form $6 k+1$ or $12 k+1$, where $k \mid m$ and $(6, k)=1$, must be a divisor of $m$. Applying (1.1), we find the following prime divisors: $13,79,157,547,1093,3319,3613,6163,6637$, $6709,36979,39829,40507,42667,45949,46957,74419,81013,85333$, 91813, 170509, 258847, 282253, 303493, 518083, 529933, 596779, $1041853,1053157,1573207,1834639,1854763,1954869,3623803$, $3641917,3669277,3856147,3944389$, 6318943, 6772039, 6806893, 7161379, 7207243, 7372093.

In cases (II) and (III), $m$ is divisible by each prime of the form $6 k+1$ or $18 k+1$, where $k$ and $m$ are as above. So in these cases $m$ is divisible by $19,127,2287,4903,5419,13723,82339,98299,101347$, 304039 , 617761, 688087, 1676827, 3736087, 4130323, 4324363, 4693267.

In case (II) we also have as divisors primes of the form $36 k+1$, and in case (III) of the form $54 k+1$. This fact gives rise to additional divisors as follows:

Case (II) : 37, 223, 1549, 4219, 4663, 4789, 9547, 10837, 25309, $27883,29527,176509,196597,197359,200467,399643,494029$, $544123,545947,1059517,1063159,1088467,1184149,1198927$, 1203019, 1235419, 1564309, 2397853, 2407141, 3265399, 3702367, 7082029, 7221439, 7274053, 7619263, 8262367, 8387839, 8647927, 9139519.

Case (III): 379, 6823, 15919, 40939, 43207, 123499, 130483, 143263, 202627, 264763, 302443, 368443, 741043, 859699, 1857859, 2018383, 2053423, 2333467, 4446759, 5030479, 5480287.

The stated divisors were found with the aid of [2]. It is easy to find still more divisors by this method. Those obtained imply the 
following lower bounds for $\bar{x}$ and $\phi(\bar{x})$ : (I) $10^{458}$; (II) $10^{586}$; (III) $10^{400}$.

\section{REFERENCES}

1. R. D. Carmichael, Note on Euler's $\phi$-function, Bull. Amer. Math. Soc. vol. 28 (1922) pp. 109-110.

2. D. N. Lehmer, List of prime numbers, Carnegie Institution Publication, no. 165.

UNIVERSITY OF VIRGINIA

\section{ON THE DARBOUX TANGENTS}

V. G. GROVE

1. Introduction. In a recent paper [1] ${ }^{1}$ Abramescu gave a metr1cal characterization of the cubic curve obtained by equating to zero the terms of the expansion of a surface $S$ at an ordinary point $O_{1}$, up to and including the terms of the third order. This cubic curve is rational and its inflexions lie on the three tangents of Darboux through $O_{1}$. In this paper we give a projective characterization of such a curve, and hence a new derivation of the tangents of Darboux. By using the method employed in this characterization to the curve of intersection of the tangent plane of the surface at $O_{1}$ with $S$, a simple characterization of the second edge of Green is found. Another application exhibits the correspondence of Moutard. Finally a new interpretation of the reciprocal of the projective normal is given in terms of the conditions of apolarity of a cubic form to a quartic form. The canonical tangent appears in a similar fashion.

Let $S$ be referred to its asymptotic curves, and let the coordinates $\left(x^{1}, x^{2}, x^{3}, x^{4}\right)$ of the generic point $O_{1}$ of $S$ be normalized so that they satisfy the system [2] of differential equations

$$
\begin{aligned}
x_{u u} & =\theta_{u} x_{u}+\beta x_{v}+p x, \\
x_{v v} & =\gamma x_{u}+\theta_{v} x_{v}+q x, \quad \theta=\log R .
\end{aligned}
$$

The line $l_{1}$ joining $O_{1}$ to $O_{4}$, whose coordinates are $x_{u v}^{i}$, is the $R$-conjugate line, and the line $l_{2}$ determined by $O_{2}, O_{3}$, whose respective coordinates are $x_{u}^{i}, x_{v}^{i}$, is the $R$-harmonic line.

If we define the local coordinates $\left(x_{1}, x_{2}, x_{3}, x_{4}\right)$ with respect to

Presented to the Society, April 26, 1947; received by the editors April 11, 1947.

${ }^{1}$ Numbers in brackets refer to the references cited at the end of the paper. 